\title{
DUNE Oscillation Physics
}

\author{
Animesh Chatterjee*t \\ University of Texas Arlington \\ E-mail: animesh.chatterjee@uta.edu
}

\begin{abstract}
The Deep Underground Neutrino Experiment (DUNE) is an international experiment currently in its design phase, for neutrino physics and proton-decay searches. Primary scientific goal of DUNE includes determining the neutrino mass hierarchy, measuring $\delta_{C P}$ with enough precision to discover leptonic $\mathrm{CP}$ violation, octant of $\theta_{23}$, precise measurement of neutrino oscillation parameters governing electron neutrino appearance and muon neutrino disappearance. The experiment will consist of a high-power, broadband neutrino beam covering a baseline of $1300 \mathrm{~km}$ from Fermilab to Sanford Underground Research Facility (SURF), a high-precision near detector and a large liquid argon time projection chamber (LArTPC) far detector which will address the major questions of neutrino physics.
\end{abstract}

The 20th International Workshop on Neutrinos (NuFact2018)

12-18 August 2018

Blacksburg, Virginia

\footnotetext{
* Speaker.

${ }^{\dagger}$ for the DUNE Collaboration
} 


\section{Introduction}

The Deep Underground Neutrino Experiment (DUNE) [1, 2] will be a world-class neutrino observatory and nucleon decay detector designed to answer fundamental questions about the nature of elementary particles and their role in the universe. The international DUNE experiment, will consist of a far detector to be located about $1.5 \mathrm{~km}$ underground at the Sanford Underground Research Facility (SURF) in South Dakota, USA, at a distance of $1300 \mathrm{~km}$ from Fermilab, and a near detector to be located at Fermilab in Illinois. The far detector will be a very large, modular liquid argon time-projection chamber (LArTPC) with a $40 \mathrm{kt}$ fiducial mass. This LAr technology will make it possible to reconstruct neutrino interactions with image-like precision and unprecedented resolution. The Long-Baseline Neutrino Facility (LBNF)([3]) will be made at Fermilab using a proton beam that will initially have a power of $1.2 \mathrm{MW}$ but can be upgraded to $2.4 \mathrm{MW}$.

DUNE plans a rich physics programme including precise measurements of neutrino oscillation parameters and determination of the neutrino mass hierarchy, whether $\mathrm{CP}$ is violated or not and the octant of $\theta_{23}$. The primary scientific program of DUNE includes as well proton-decay searches and neutrino astrophysics.

\section{Sensitivities to neutrino oscillation physics}

Precision neutrino oscillation measurements are the major scientific program of the DUNE experiment. DUNE plans to carry out a detailed study of neutrino mixing, resolve the neutrino mass ordering and search for $\mathrm{CP}$ violation in the lepton sector by studying the oscillation spectra of $v_{\mu}\left(\overline{v_{\mu}}\right)$ and $v_{e}\left(\bar{v}_{e}\right)$. The electron neutrino appearance probability is sensitive to the value of the $\delta$ phase, the neutrino mass ordering and $\theta_{23}$ octant through $\sin ^{2} \theta_{23}$.

The experimental sensitivities presented here are estimated using GLoBES[4, 5]. GLoBES takes neutrino beam fluxes, cross sections and a detector-response parameterization as inputs. The neutrino cross-section are taken from GENIE[6] and neutrino oscillation parameters taken from NuFIT[7] data. Sensitivity to the CP violation, mass ordering, $\theta_{23}$ octant and neutrino oscillation parameters are obtained by simultanious fitting of the four neutrino spectra $v_{\mu} \rightarrow v_{\mu}, v_{\mu} \rightarrow v_{e}$, $\overline{v_{\mu}} \rightarrow \overline{v_{\mu}}, \overline{v_{\mu}} \rightarrow \overline{v_{e}}$.

The mass hierarchy sensitivity of DUNE for exposures of seven and ten years is shown in left panel of Figure 1 for the case of normal mass ordering. It is clear that even with 7 years of exposure, DUNE experiment can determine MH overwhelmingly for all values of $\delta_{C P}$. Right hand panel of Figure 1 shows the evolution of the sensitivity to the $\mathrm{MH}$ determination as a function of years of operation, for all the $\delta_{C P}$ parameter space. Sensitivity to the CP violation for exposures of seven and ten years for the case of normal mass ordering is shown in left panel of Figure 2, and the significance with which CP violation can be determined for $75 \%$ and $50 \%$ of $\delta_{C P}$ values and for $\delta_{C P}=-\pi / 2$ shown in right panel of Figure 2. Sensitivity to $\theta_{23}$ octant as a function of the true value of $\sin ^{2} \theta_{23}$ is shown in left panel of Figure 3 for the exposure of seven (green) and ten (orange) years. The yellow band shows the $90 \%$ C.L. range of allowed values of $\sin ^{2} \theta_{23}$ from NuFIT data. Sensitivity to the $\delta_{C P}$ resolution as a function of running time (right) for the normal mass hierarchy is shown in right panel of Figure 3.

\section{Conclusion}

The Deep Underground Neutrino Experiment (DUNE) will be the leading edge neutrino 

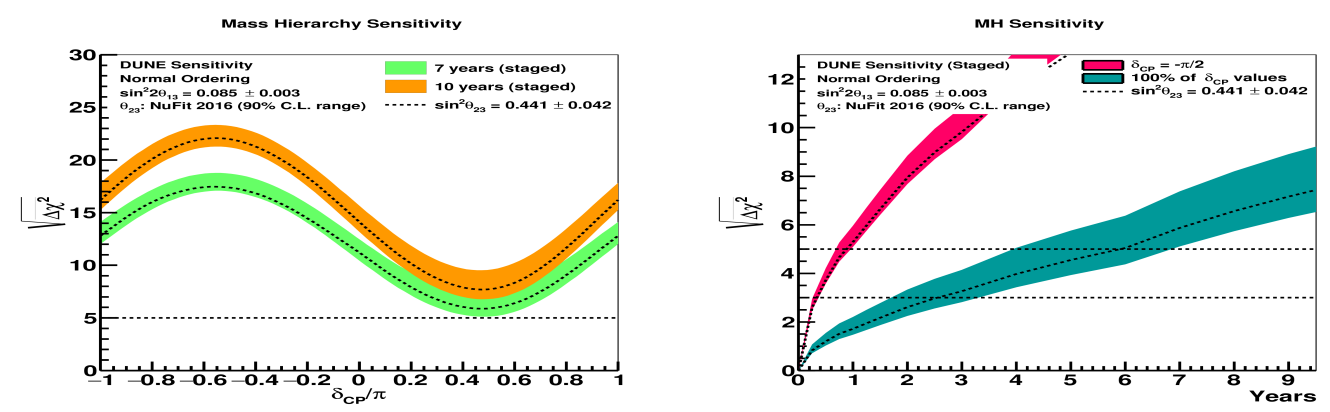

Figure 1: The sensitivity of the mass hierarchy discrimination is plotted as a function of the unknown value of $\delta_{C P}$ for exposures of seven and ten years (left) and the significance for a true value of $\delta_{C P}=-\pi / 2$ as a function of years of running under the staging plan described in the text (right). The shaded regions represent the range in sensitivity corresponding to different true values of $\theta_{23}$.
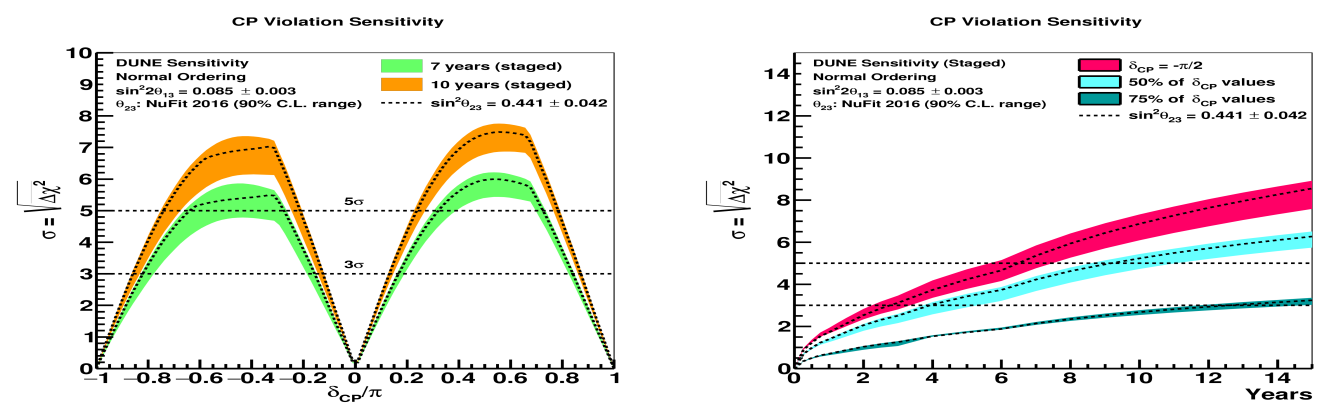

Figure 2: Sensitivity to $\mathrm{CP}$ violation, defined as $\delta_{C P} \neq 0$ or $\pi$, as a function of $\delta_{C P}$ (the normal mass hierarchy is assumed) and the significance with which CP violation can be determined for $75 \%$ and $50 \%$ of $\delta_{C P}$ values and for $\delta_{C P}=-\pi / 2$ (right).
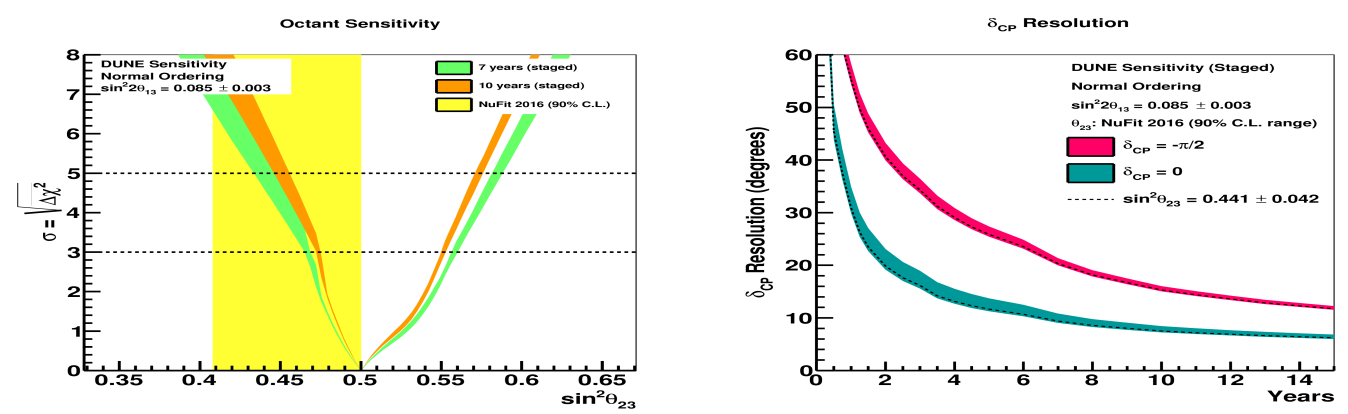

Figure 3: Sensitivity to $\theta_{23}$ octant as a function of the true value of $\sin ^{2} \theta_{23}$ (left) and $\delta_{C P}$ resolution as a function of running time (right) for the normal mass hierarchy.

experiment. It utilizes a mega-watt class proton accelerator (with beam power of up to $2.4 \mathrm{MW}$ ), a massive (40 kt) liquid argon time-projection chamber (LArTPC) far detector (FD), and a high precision near neutrino detector (ND). Due to its long baseline of $1300 \mathrm{~km}$, neutrino oscillations between the near and far detectors will be significantly altered by matter effects. These features will enable DUNE to search for CP violation in neutrinos, measure $\delta_{C P}$, and resolve the neutrino mass hierarchy and the $\theta_{23}$ octant in a single experiment. DUNE will also contribute significantly for the precision measurement of the all other oscillation parameters. 


\section{References}

[1] R. Acciarri et al. [DUNE Collaboration], arXiv:1601.05471 [physics.ins-det].

[2] R. Acciarri et al. [DUNE Collaboration], arXiv:1512.06148 [physics.ins-det].

[3] J. Strait et al. [DUNE Collaboration], arXiv:1601.05823 [physics.ins-det].

[4] P. Huber, M. Lindner and W. Winter, Comput. Phys. Commun. 167, 195 (2005) doi:10.1016/j.cpc.2005.01.003 [hep-ph/0407333].

[5] P. Huber, J. Kopp, M. Lindner, M. Rolinec and W. Winter, Comput. Phys. Commun. 177, 432 (2007) doi:10.1016/j.cpc.2007.05.004 [hep-ph/0701187].

[6] C. Andreopoulos et al., Nucl. Instrum. Meth. A 614, 87 (2010) doi:10.1016/j.nima.2009.12.009 [arXiv:0905.2517 [hep-ph]].

[7] I. Esteban, M. C. Gonzalez-Garcia, M. Maltoni, I. Martinez-Soler and T. Schwetz, JHEP 1701, 087 (2017) doi:10.1007/JHEP01(2017)087 [arXiv:1611.01514 [hep-ph]]. 